\title{
Lower bounds for a certain class of error functions
}

by

\section{J. Herzog and P. R. Smith (Frankfurt)}

1. Introduction. An arithmetical function $f$ that does not deviate too largely from the identity function $I: n \mapsto n$ frequently satisfies an asymptotic relation

$$
\sum_{n \leq x} f(n)=C_{f} x^{2}+R_{f}(x),
$$

in which the error term $R_{f}(x)$ is the primary object of interest.

A quite thoroughly investigated example is provided by Euler's totient $\varphi$. For instance, A. Walfisz's [17] well known upper bound

$$
R_{\varphi}(x)=\sum_{n \leq x} \varphi(n)-\frac{3}{\pi^{2}} x^{2} \ll x(\log x)^{2 / 3}(\log \log x)^{4 / 3}
$$

has superseded F. Mertens' elementary estimate [12]

$$
R_{\varphi}(x) \ll x \log x,
$$

and in the opposite direction there are the results due to S. S. Pillai and S. D. Chowla [14]

$$
R_{\varphi}(x)=\Omega(x \log \log \log x)
$$

and P. Erdős and H. N. Shapiro [4]

$$
R_{\varphi}(x)=\Omega_{ \pm}(x \log \log \log \log x) .
$$

Subsequently J. H. Proschan [15] applied the techniques of [4] and [14] to obtain $\Omega$-results for the remainder term $R_{f}(x)$ corresponding to arithmetical functions $f=I *(\mu \cdot g)$, where $\mu$ is the Möbius function and $g$ is a positive integer valued completely multiplicative function that satisfies certain growth conditions.

In this paper we will show how a method that has recently been used by H. L. Montgomery [13] to improve (1.1) and (1.2) to

$$
R_{\varphi}(x)=\Omega_{ \pm}(x \sqrt{\log \log x})
$$


can be extended to a class of arithmetical functions that is considerably larger than that which was treated in [15].

Moreover, our estimates are as a rule much sharper than Proschan's, typically improving his $\Omega_{ \pm}(x \log \log \log \log x)$ to $\Omega_{ \pm}\left(x(\log \log x)^{\delta}\right)$ for an appropriate positive constant $\delta=\delta(f)$.

Our results are applicable to many generalizations of Euler's $\varphi$-function, e.g. the totients of Schemmel and Nagell (cf. [16]) and the function $\varphi_{F}$ defined with respect to an irreducible polynomial $F \in \mathbb{Z}[x]$ by

$$
\varphi_{F}(n):=n \prod_{p \mid n}\left(1-\frac{\varrho(p)}{p}\right)
$$

where $\varrho(p)$ denotes the number of zeros of $F(x)(\bmod p)$.

2. Definitions and statement of main results. The members of the class of functions that we investigate are of the form $f=I * h$, where $h$ is an arithmetical function that has certain properties in common with the Möbius function.

However, the similarity between $h$ and $\mu$ need not be too close, since $h$ is allowed to be unbounded, for example. The precise conditions that are to be fulfilled by $h$ are summarized in the following

Definition 2.1. For real $r \geq 0$ and a positive integer $k$ the class $\mathcal{C}(r, k)$ consists of all real-valued multiplicative arithmetical functions $h$ which satisfy

(2.3) there exists an integer $B \geq 1$ such that $h\left(p^{i}\right)=0$ for primes $p$ not dividing $B$ and $1 \leq i<k$;

(2.4) if $n$ is a $k$-full integer then $h(n)=\mu(\alpha(n))|h(n)|$, where $\alpha(n):=$ $\prod_{p \mid n} p$ is the squarefree kernel of $n$;

(2.5) the series $\sum_{p}\left|h\left(p^{k}\right)\right| p^{-k}$ diverges;

(2.6) the series $\sum_{p}\left|h\left(p^{k}\right)\right|^{2} p^{-2 k}$ converges.

Remarks. (a) Throughout the letter $p$ denotes a prime.

(b) Note that (2.1) implies that $\sum_{n \geq 1} h(n) n^{-1-\varepsilon}$ converges absolutely for every $\varepsilon>0$.

(c) The Möbius function is in $\mathcal{C}(0,1)$. 
Our primary result is

TheOREm 2.2. Let $f:=I * h$ where $h \in \mathcal{C}(r, k)$. Suppose there is a monotonically decreasing function $\xi$, defined for $x>0$, which has the following properties:

$$
\sup _{y>x}\left|\sum_{x<n \leq y} \frac{h(n)}{n}\right| \leq \xi(x) \quad(x>0) ;
$$

$$
\xi(x)(\log x)^{r} \text { is decreasing for sufficiently large } x \text { and }
$$

$$
\lim _{x \rightarrow \infty} \xi(x)(\log x)^{r}=0 ;
$$

Furthermore, assume there is an integer $M \geq 3$ for which the congruence $x^{k} \equiv-1(\bmod M)$ has $\Delta \varphi(M) \geq 1$ solutions $(\bmod M)$ and such that for integers a, relatively prime to $M$,

$$
\sum_{\substack{p \leq x \\ p \equiv a(\bmod M)}}\left|h\left(p^{k}\right)\right| p^{-k}=\frac{1}{\varphi(M)} \Theta(x)+O(1)
$$

where

$$
\Theta(x):=\sum_{p \leq x}\left|h\left(p^{k}\right)\right| p^{-k}
$$

Set

$$
L(x):=\left((\log x)^{r} \cdot \xi\left(x(\log x)^{-r}\right)\right)^{-1} .
$$

Then we have

$$
\sum_{n \leq x} \frac{f(n)}{n}=c(h) x+E(x)
$$

where

$$
E(x) \ll(\log x)^{r+1}
$$

and

$$
E(x)=\Omega_{ \pm}\left(\exp \left(\Delta \cdot \Theta\left((2 \Delta k)^{-1} \log L(\sqrt{x})\right)\right)\right) .
$$

In most cases the conclusion of the theorem carries over to the perhaps more natural error term

$$
R(x)=\sum_{n \leq x} f(n)-\frac{1}{2} c(h) x^{2} .
$$

This is the subject of the first of the next two corollaries, for which we retain the notation and assumptions of Theorem 2.2. 
Corollary 2.3. We have

$$
R(x) \ll x(\log x)^{r+1}
$$

and, if additionally $\xi(x) \log x \ll 1$, then

$$
R(x)=\Omega_{ \pm}\left(x \cdot \exp \left(\Delta \cdot \Theta\left((2 \Delta k)^{-1} \log L(\sqrt{x})\right)\right)\right) .
$$

Corollary 2.4. If $\lim _{x \rightarrow \infty} \xi(x) \log x=0$ then

$$
\sum_{n \leq x} E(n) \sim \frac{1}{2}(c(h)-b(h)) x
$$

and

$$
\sum_{n \leq x} R(n) \sim \frac{1}{4} c(h) x^{2}
$$

where

$$
b(h):=\sum_{n=1}^{\infty} \frac{h(n)}{n} .
$$

3. Proof of Theorem 2.2. It follows from $f=I * h$ and Abel's inequality (cf. [11], Satz 140) that

$$
\begin{aligned}
E(x) & =-x \sum_{n>x} h(n) n^{-2}-\sum_{n \leq x} \frac{h(n)}{n}\left\{\frac{x}{n}\right\} \\
& =-\sum_{n \leq x} \frac{h(n)}{n}\left\{\frac{x}{n}\right\}+O(\xi(x)) .
\end{aligned}
$$

Here $\{t\}$ denotes the fractional part of the real number $t$.

From (3.1) we deduce that for all positive $x$ and $y$

$$
E(x)=-\sum_{n \leq y} \frac{h(n)}{n}\left\{\frac{x}{n}\right\}+O(\xi(x))+O\left(\frac{x}{y} \xi(y / 2)\right) .
$$

This is because for $y \leq x$ we have

$$
\begin{aligned}
\left|\sum_{y<n \leq x} \frac{h(n)}{n}\left\{\frac{x}{n}\right\}\right| & =\left|\sum_{1 \leq k \leq x / y} \sum_{\substack{x /(k+1)<n \leq x / k \\
n>y}} \frac{h(n)}{n}\left\{\frac{x}{n}\right\}\right| \\
& \leq \sum_{k \leq x / y} \xi(x /(k+1)) \leq \frac{x}{y} \xi(y / 2),
\end{aligned}
$$

and for $y>x$

$$
\left|\sum_{x<n \leq y} \frac{h(n)}{n}\left\{\frac{x}{n}\right\}\right| \leq \xi(x) .
$$


Following Montgomery [13] we introduce the function

$$
s(t):= \begin{cases}\frac{1}{2}-\{t\} & \text { if } t \notin \mathbb{Z}, \\ 0 & \text { if } t \in \mathbb{Z}\end{cases}
$$

into formula (3.2) and use the convergence of $\sum_{n=1}^{\infty} h(n) n^{-1}$ to obtain for $y>0$ and nonintegral $x>0$

$$
E(x)=\sum_{n \leq y} \frac{h(n)}{n} s\left(\frac{x}{n}\right)+O\left(\frac{x}{y} \xi(y / 2)\right)+O(1) .
$$

For natural numbers $d, q$ and $N$ and nonintegral $\beta, 0<\beta<q$, we have (cf. [13], Lemma 3)

$$
\sum_{n=1}^{N} s\left(\frac{n q+\beta}{d}\right)=(d, q) s\left(\frac{\beta}{(d, q)}\right) \frac{N}{d}+O(d),
$$

which along with (3.3) and (2.1) yields (upon inverting the order of summation) for $y>0$

$$
\begin{aligned}
\sum_{n=1}^{N} E(n q+\beta)= & N \sum_{d \leq y} \frac{h(d)}{d^{2}}(d, q) s\left(\frac{\beta}{(d, q)}\right)+O(N) \\
& +O\left(y(\log y)^{r}\right)+O\left(N^{2} q y^{-1} \xi(y / 2)\right) .
\end{aligned}
$$

The above formula (3.4) suggests a closer investigation of

$$
\Sigma(y, q, \beta):=\sum_{d \leq y} \frac{h(d)}{d^{2}}(d, q) s\left(\frac{\beta}{(d, q)}\right) .
$$

Since $h$ is multiplicative and each natural number $d$ may be written uniquely as $d=u v$ where $\alpha(u) \mid q$ and $(v, q)=1$, we have

$$
\Sigma(y, q, \beta)=\sum_{\substack{u \leq y \\ \alpha(u) \mid q}} \frac{h(u)}{u^{2}}(u, q) s\left(\frac{\beta}{(u, q)}\right) \sum_{\substack{v \leq y / u \\(v, q)=1}} \frac{h(v)}{v^{2}} .
$$

For the sake of convenience set

$$
\Phi_{q}:=\sum_{\substack{v \geq 1 \\(v, q)=1}} h(v) v^{-2}
$$

and note that (2.1) and partial summation imply that

$$
\Phi_{q}=\sum_{\substack{v \leq y / u \\(v, q)=1}} h(v) v^{-2}+O\left(\frac{u}{y}(\log y)^{r}\right) .
$$


Since (again by partial summation)

$$
\sum_{\substack{u \leq y \\ \alpha(u) \mid q}} \frac{|h(u)|}{u}(u, q) \leq q \sum_{u \leq y} \frac{|h(u)|}{u} \ll q(\log y)^{r+1},
$$

formulas (3.6) and (3.7) give

$$
\Sigma(y, q, \beta)=\Phi_{q} \sum_{\substack{u \leq y \\ \alpha(u) \mid q}} \frac{h(u)}{u^{2}}(u, q) s\left(\frac{\beta}{(u, q)}\right)+O\left(\frac{q}{y}(\log y)^{2 r+1}\right) .
$$

Recall (cf. (2.3)) the existence of an integer $B$ such that $h\left(p^{i}\right)=0$ whenever $1 \leq i<k$ and $(p, B)=1$, and choose for a given $y \geq 1$ a squarefree natural number $Q$ satisfying

$$
(Q, B)=1 \quad \text { and } \quad q:=Q^{k} \leq y .
$$

Taking into account that $h(u)=0$ whenever $\alpha(u) \mid q$, unless $u$ is $k$-full, we may parametrize the integers $u$ in (3.8) by $u=a^{k} b$, where $a$ is a (necessarily squarefree) divisor of $Q$ and $\alpha(b) \mid a$. Thus we obtain

$$
\begin{aligned}
\Sigma(y, q, \beta)= & \Phi_{q} \sum_{a \mid Q} \frac{\mu(a)}{a^{k}} s\left(\frac{\beta}{a^{k}}\right) \sum_{\substack{b \leq y / a^{k} \\
\alpha(b) \mid a}} \frac{\left|h\left(a^{k} b\right)\right|}{b^{2}} \\
& +O\left(\frac{q}{y}(\log y)^{2 r+1}\right),
\end{aligned}
$$

where we have used (2.4).

Now set $m:=\Delta \varphi(M)$ and denote by $r_{1}, \ldots, r_{m}$ representatives of the distinct residue classes $x(\bmod M)$ which satisfy $x^{k} \equiv-1(\bmod M)$.

Let $t \geq t_{0}$ be a real parameter, and define

$$
Q:=\prod_{\substack{p \leq t \\(p, B)=1 \\ p \equiv r_{1}, \ldots, r_{m}(\bmod M)}} p .
$$

Determine $N$ as the smallest natural number such that

$$
N \geq 2 \quad \text { and } \quad L(N-1)<q=Q^{k} \leq L(N) .
$$

As (2.8) ensures that $\lim _{x \rightarrow \infty} L(x)=\infty, N$ is well defined provided $t_{0}$ is large enough. With

$$
y:=2 N(\log N)^{-r}
$$

it follows from (2.9) that $q \leq y$ for large $t$, i.e. (3.9) is satisfied, and thus 
(3.4), (3.5) and (3.10) may be combined to yield

$$
\sum_{n \leq N} E(n q+\beta)=N \Phi_{q} \sum_{a \mid Q} \frac{\mu(a)}{a^{k}} s\left(\frac{\beta}{a^{k}}\right) \sum_{\substack{b \leq y / a^{k} \\ \alpha(b) \mid a}} \frac{\left|h\left(a^{k} b\right)\right|}{b^{2}}+O(N) .
$$

The influence of the factor $\Phi_{q}$ on the size and the sign of the right side of (3.14) is negligible since

$$
\left|\Phi_{q}\right| \geq\left|\sum_{n \geq 1} \frac{h(n)}{n^{2}}\right|\left(\sum_{n \geq 1} \frac{|h(n)|}{n^{2}}\right)^{-1},
$$

and the sign of $\Phi_{q}$ is constant for large $t$, as one sees upon consideration of the relevant Euler factors $\sum_{i \geq 0} h\left(p^{i}\right) p^{-2 i}$. Thus without loss of generality we may suppose that $\Phi_{q}$ remains larger than a fixed positive constant.

To obtain the $\Omega_{+}$-result for $E(x)$ we restrict the parameter $t$ to the range of values for which $\mu(Q)=1$. With $\beta=q / M$ the conditions $0<\beta<q$ and $\beta \notin \mathbb{Z}$ are trivially satisfied.

If $a$ divides $Q$ then

$$
\frac{\beta}{a^{k}}=\left(\frac{Q}{a}\right)^{k} \frac{1}{M} \quad \text { and } \quad\left(\frac{Q}{a}\right)^{k} \equiv \mu(a)(\bmod M),
$$

which implies that

$$
\mu(a) s\left(\beta / a^{k}\right)=1 / 2-1 / M \geq 1 / 6 .
$$

Hence we deduce from (3.14) that

$$
\begin{aligned}
\sum_{n \leq N} E(n q+\beta) & \gg N \sum_{a \mid Q} a^{-k} \sum_{\substack{b \leq y / a^{k} \\
\alpha(b) \mid a}}\left|h\left(a^{k} b\right)\right| b^{-2}+O(N) \\
& \gg N \sum_{a \mid Q}\left|h\left(a^{k}\right)\right| a^{-k}+O(N),
\end{aligned}
$$

whence

$$
\sum_{n \leq N} E(n q+\beta) \gg N \prod_{p \mid Q}\left(1+\left|h\left(p^{k}\right)\right| p^{-k}\right)+O(N) .
$$

Here we have used $a^{k} \leq Q^{k}=q \leq y$ to estimate from below each sum over $b$ by $\left|h\left(a^{k}\right)\right|$.

Since $1+x \geq\left(1-x^{2}\right) e^{x}$ for $x \geq 0$, and in view of (2.6), (2.10), (2.11) and (3.11), we have

$$
\prod_{p \mid Q}\left(1+\left|h\left(p^{k}\right)\right| p^{-k}\right) \gg \exp \left(\sum_{p \mid Q}\left|h\left(p^{k}\right)\right| p^{-k}\right) \gg \exp (\Delta \cdot \Theta(t)) .
$$


The prime number theorem for arithmetic progressions gives

$$
\log Q=\sum_{\substack{p \leq t \\ p \equiv r_{1}, \ldots, r_{m}(\bmod M)}} \log p+O(1) \sim \Delta t,
$$

and therefore

$$
\log \log Q=\log t+\log \Delta+o(1) .
$$

Moreover, (2.9), (2.12) and (3.12) show that $q=Q^{k} \sim L(N)$, whence

$$
\log \log Q=\log \log L(N)-\log k+o(1) .
$$

Combining (3.17) and (3.18) we obtain

$$
t \sim(k \Delta)^{-1} \log L(N),
$$

and thus by (3.15) and (3.16)

$$
\sum_{n \leq N} E(n q+\beta) \gg N \exp \left(\Delta \cdot \Theta\left(\frac{1+o(1)}{k \Delta} \log L(N)\right)\right) .
$$

The function $L^{*}(x)$ defined by

$$
\left(L^{*}(x)\right)^{-1}:=\left(\log \left(x(\log x)^{-r}\right)\right)^{r} \cdot \xi\left(x(\log x)^{-r}\right)
$$

is increasing for sufficiently large $x$ and satisfies

$$
\log L^{*}(x)=\log L(x)+o(1) \quad(x \rightarrow \infty) .
$$

Since $\Theta(x+O(1))=\Theta(x)+o(1)$ it follows from (3.19) that

$$
\sum_{n \leq N} E(n q+\beta) \gg N \exp \left(\Delta \cdot \Theta\left((2 \Delta k)^{-1} \log L^{*}(N)\right)\right) .
$$

As $n q+\beta \leq N^{2}(1 \leq n \leq N)$ for large $t$, the relation

$$
E(x)=o\left(\exp \left(\Delta \cdot \Theta\left((2 \Delta k)^{-1} \log L^{*}(\sqrt{x})\right)\right)\right)
$$

or its equivalent

$$
E(x)=o\left(\exp \left(\Delta \cdot \Theta\left((2 \Delta k)^{-1} \log L(\sqrt{x})\right)\right)\right)
$$

would imply

$$
\sum_{n \leq N} E(n q+\beta)=o\left(N \exp \left(\Delta \cdot \Theta\left((2 \Delta k)^{-1} \log L^{*}(N)\right)\right)\right),
$$

which contradicts (3.20). This proves the $\Omega_{+}$-part of (2.15).

The same argument may be used to obtain the corresponding $\Omega_{-}$-result: one need only require $t$ in (3.11) to run through values for which $\mu(Q)=-1$.

The estimate $E(x) \ll(\log x)^{r+1}$ follows immediately from (2.1), (3.1) and partial summation. This completes the proof of the theorem. 
Proof of Corollary 2.3. From $f=I * h$ we infer

$$
\begin{aligned}
R(x)= & -\frac{1}{2} x^{2} \sum_{n>x} h(n) n^{-2}-x \sum_{n \leq x} \frac{h(n)}{n}\left\{\frac{x}{n}\right\}+\frac{1}{2} x \sum_{n \leq x} \frac{h(n)}{n} \\
& +\frac{1}{2} \sum_{n \leq x} h(n)\left(\left\{\frac{x}{n}\right\}^{2}-\left\{\frac{x}{n}\right\}\right) .
\end{aligned}
$$

Therefore (3.1) and the convergence of $\sum_{n \geq 1} h(n) \cdot n^{-1}$ yield

$$
R(x)=x E(x)+O(x)+\frac{1}{2} \sum_{n \leq x} h(n)\left(\left\{\frac{x}{n}\right\}^{2}-\left\{\frac{x}{n}\right\}\right),
$$

and consequently $R(x) \ll x(\log x)^{r+1}$ in view of (2.1) and (2.14).

Moreover, (2.1) and the assumption that $\xi(x) \ll(\log x)^{-1}$ yield

$$
\begin{aligned}
\mid \sum_{n \leq x} h(n) & \left(\left\{\frac{x}{n}\right\}^{2}-\left\{\frac{x}{n}\right\}\right) \mid \\
& \leq \sum_{n \leq \sqrt{x}}|h(n)|+\left|x \int_{\sqrt{x}}^{x} \sum_{\sqrt{x}<n \leq t} h(n)\left(2\left\{\frac{x}{t}\right\}-1\right) t^{-2} d t\right| \\
& \ll x^{3 / 4}+x \xi(\sqrt{x}) \log x \ll x,
\end{aligned}
$$

since Abel's inequality gives

$$
\left|\sum_{\sqrt{x}<n \leq t} \frac{h(n)}{n} n\right| \leq t \xi(\sqrt{x}) .
$$

Proof of Corollary 2.4. A comparison of formulas (3.1) and (3.21) shows that the assumption $\xi(x)=o(1 / \log x)$ implies

$$
R(x)=x E(x)+\frac{1}{2} b(h) x+o(x) .
$$

Therefore (2.20) follows from (2.19) by partial summation. To obtain (2.19) one may use the standard approach of Pillai and Chowla [14].

4. Applications. In some of the applications of Theorem 2.2 and its corollaries it is important to have estimates for sums involving iterates of the Möbius function.

Lemma 4.1. For $d \geq 2$ let $\mu_{d}:=\mu_{d-1} * \mu$, where $\mu_{1}:=\mu$. Then for every $d \geq 1$ there is a positive constant $c_{d}$ for which

$$
\sum_{n \leq x} \mu_{d}(n) n^{-1} \ll_{d} \exp \left(-c_{d} \sqrt{\log x}\right) .
$$


Proof. By induction. The case $d=1$ is the prime number theorem. Since $\mu_{d}\left(p^{j}\right)=(-1)^{j}\left(\begin{array}{l}d \\ j\end{array}\right)$, it follows that

$$
\sum_{n \leq x}\left|\mu_{d}(n)\right| n^{-1} \leq \prod_{p \leq x}\left(\sum_{j \geq 0}\left|\mu_{d}\left(p^{j}\right)\right| p^{-j}\right) \ll(\log x)^{d} .
$$

The inductive step is therefore a consequence of the identity (cf. [1], Thm. 3.17),

$$
\begin{aligned}
\sum_{n \leq x} \mu_{d}(n) n^{-1}= & \sum_{n \leq \sqrt{x}} \mu_{d-1}(n) n^{-1} \sum_{m \leq x / n} \mu(m) m^{-1} \\
& +\sum_{n \leq \sqrt{x}} \mu(n) n^{-1} \sum_{m \leq x / n} \mu_{d-1}(m) m^{-1} \\
& -\sum_{n \leq \sqrt{x}} \mu_{d-1}(n) n^{-1} \sum_{n \leq \sqrt{x}} \mu(n) n^{-1} .
\end{aligned}
$$

Our first application deals with Nagell's totient, which is defined for every natural $j$ by

$$
\theta(j, n):=n \prod_{p \mid n}\left(1-\frac{\varepsilon(j, p)}{p}\right)
$$

where

$$
\varepsilon(j, p):= \begin{cases}1 & \text { if } p \mid j \\ 2 & \text { if }(p, j)=1\end{cases}
$$

TheOREM 4.2. For every positive integer $j$ let

$$
\gamma(j):=\frac{1}{2} \prod_{p \mid j}\left(p^{2}-1\right)\left(p^{2}-2\right)^{-1} \prod_{p}\left(1-2 p^{-2}\right) .
$$

Then

$$
\sum_{n \leq x} \theta(j, n)=\gamma(j) x^{2}+R_{j}(x)
$$

where

$$
R_{j}(x) \ll x(\log x)^{2}
$$

and

$$
R_{j}(x)=\Omega_{ \pm}(x \log \log x) .
$$

Proof. Write $\theta(j, n)=I * h_{j}(n)$, where $h_{j}(p):=-\varepsilon(j, p)$ and $h_{j}\left(p^{\alpha}\right):=$ 0 whenever $\alpha \geq 2$. A standard argument (cf. [5], Thm. 2) shows that

$$
\sum_{n \leq x}\left|h_{j}(n)\right| \ll \frac{x}{\log x} \prod_{p \leq x}\left(1+\left|h_{j}(p)\right| p^{-1}\right) \ll x \log x,
$$

whence $h_{j} \in \mathcal{C}(1,1)$. 
In order to estimate $\sum_{x<n \leq y} h_{j}(n) n^{-1}$, we factorize $h_{j}$ as $h_{j}=\mu_{2} * A_{j}$. The Euler product

$$
\prod_{p}\left(\sum_{\nu \geq 0} A_{j}\left(p^{\nu}\right) p^{-\nu s}\right)=\prod_{p \mid j} \frac{1-p^{-s}}{1-2 p^{-s}} \prod_{p}\left(1-\left(p^{s}-1\right)^{-2}\right)
$$

converges absolutely in $\operatorname{Re} s>1 / 2$, and thus $\sum_{n \geq 1} A_{j}(n) n^{-1 / 2-\varepsilon}$ converges absolutely for every $\varepsilon>0$.

Therefore by Lemma 4.1

$$
\begin{aligned}
\sum_{n \leq x} h_{j}(n) n^{-1}= & \sum_{n \leq \sqrt{x}} A_{j}(n) n^{-1} \sum_{m \leq x / n} \mu_{2}(m) m^{-1} \\
& +\sum_{\sqrt{x}<n \leq x} A_{j}(n) n^{-1} \sum_{m \leq x / n} \mu_{2}(m) m^{-1} \\
\ll & \exp (-c \sqrt{\log x})
\end{aligned}
$$

for some positive constant $c=c(j)$. Hence there exist constants $c_{1}=c_{1}(j)$ and $c_{2}=c_{2}(j)$ such that for $x>0$ we have

$$
\sup _{y>x}\left|\sum_{x<n \leq y} h_{j}(n) n^{-1}\right| \leq c_{1} \exp \left(-c_{2} \sqrt{\log (1+x)}\right)=: \xi_{j}(x) .
$$

Obviously $\xi_{j}(x)$ satisfies the assumptions of Corollary 2.3. Furthermore,

$$
\Theta_{j}(x)=\sum_{p \leq x}\left|h_{j}(p)\right| p^{-1}=2 \log \log x+O(1),
$$

and since $k=1$ we may take $M=3$ (which implies $\Delta=1 / 2$ ), so (2.10) is fulfilled. As $\log L(\sqrt{x}) \gg \sqrt{\log x}$, we have

$$
\Delta \cdot \Theta_{j}\left((2 \Delta k)^{-1} \log L(\sqrt{x})\right) \geq \log \log \log x+O(1)
$$

and Theorem 4.2 follows from Corollary 2.3.

In the same way we may also deal with Schemmel's totient, which is a multiplicative function defined for every natural $j$ by

$$
\Phi_{j}\left(p^{\alpha}\right):= \begin{cases}0 & \text { if } p \leq j, \\ p^{\alpha}(1-j / p) & \text { if } p>j .\end{cases}
$$

Theorem 4.3. For natural $j$ let

$$
\lambda(j):=\frac{1}{2} \prod_{p \leq j}\left(1-p^{-1}\right) \prod_{p>j}\left(1-j p^{-2}\right) .
$$

Then

$$
\sum_{n \leq x} \Phi_{j}(n)=\lambda(j) x^{2}+R_{j}(x)
$$

where

$$
R_{j}(x) \ll x(\log x)^{j}
$$


and

$$
R_{j}(x)=\Omega_{ \pm}\left(x(\log \log x)^{j / 2}\right) .
$$

Pr o of. In this case we have $\Phi_{j}=I * h_{j}$, with

$$
h_{j}\left(p^{\alpha}\right):= \begin{cases}0 & \text { if } \alpha \geq 2, \\ -p & \text { if } \alpha=1 \text { and } p \leq j, \\ -j & \text { if } \alpha=1 \text { and } p>j .\end{cases}
$$

It is readily verified that $h_{j} \in \mathcal{C}(j-1,1)$. As before we factor $h_{j}$ as $h_{j}=$ $\mu_{j} * B_{j}$, where $\sum_{n \geq 1} B_{j}(n) n^{-1 / 2-\varepsilon}$ converges absolutely for every $\varepsilon>0$. In view of Lemma 4.1 we then obtain

$$
\sup _{y>x}\left|\sum_{x<n \leq y} h_{j}(n) n^{-1}\right| \ll \exp (-c \sqrt{\log x})
$$

for an appropriate constant $c=c(j)>0$.

Again we may choose $M=3$; since

$$
\Delta \cdot \Theta_{j}(x)=\frac{1}{2} \sum_{p \leq x}\left|h_{j}(p)\right| p^{-1}=(j / 2) \log \log x+O(1)
$$

and $\log L(\sqrt{x}) \gg \sqrt{\log x}$, Corollary 2.3 yields the theorem.

As a further application of the results of Section 2 we will consider the multiplicative function $\varphi_{F}$ defined with respect to an irreducible polynomial $F \in \mathbb{Z}[x]$ of degree $g \geq 1$ by

$$
\varphi_{F}(n):=n \prod_{p \mid n}\left(1-\varrho_{F}(p) / p\right)
$$

where $\varrho_{F}(p)$ is the number of zeros of $F(x)(\bmod p)$. The verification of the premises of Theorem 2.2 and Corollary 2.3 is somewhat more arduous than in the first two examples and will be taken care of in a series of lemmas.

In the sequel $F(x)=a_{g} x^{g}+\ldots+a_{1} x+a_{0} \in \mathbb{Z}[x]$ denotes a fixed irreducible polynomial of degree $g \geq 1$. Furthermore, let $K$ be a splitting field of $F(x) / \mathbb{Q}$ and $\eta \in K$ a fixed zero of $F$. If we write $\varphi_{F}=I * h_{F}$, then

$$
h_{F}\left(p^{\alpha}\right)= \begin{cases}-\varrho_{F}(p) & \text { if } \alpha=1, \\ 0 & \text { if } \alpha \geq 2 .\end{cases}
$$

From Erdős ([3], Lemma 7) it follows that

$$
\Theta_{F}(x)=\sum_{p \leq x}\left|h_{F}(p)\right| p^{-1}=\sum_{p \leq x} \varrho_{F}(p) p^{-1}=\log \log x+O(1),
$$

and thus (cf. [5], Thm. 2)

$$
\sum_{n \leq x}\left|h_{F}(n)\right| \ll \frac{x}{\log x} \prod_{p \leq x}\left(1+\varrho_{F}(p) / p\right) \ll x,
$$

so that $h_{F} \in \mathcal{C}(0,1)$. 
Lemma 4.4. For $p$ unramified in $\mathbb{Q}(\eta)$, if $a_{g}$ and the discriminant $\Delta\left(1, \eta, \ldots, \eta^{g-1}\right)$ are $p$-adic units, then $\varrho_{F}(p)$ is the number of prime divisors of $p$ of degree one in $\mathbb{Q}(\eta)$.

Proof. For $a_{g}=1$ the proof is well known (cf. [2], pp. 212-213). The general case is an immediate consequence of [7] (Thm. 7.6 and Prop. 7.7).

Lemma 4.5. There are positive constants $c_{1}=c_{1}(F)$ and $c_{2}=c_{2}(F)$ such that for $x>0$

$$
\sup _{y>x}\left|\sum_{x<n \leq y} h_{F}(n) n^{-1}\right| \leq c_{1} \exp \left(-c_{2}(\log (1+x))^{1 / 12}\right) .
$$

Pr o of. By Lemma 4.4 there exists a positive integer $D$ for which $\varrho_{F}(p)$ is the number of prime divisors of $p$ of degree one in $\mathbb{Q}(\eta)$, whenever $p$ does not divide $D$.

Let $\zeta_{F}(s):=\prod_{\mathfrak{p}}\left(1-N(\mathfrak{p})^{-s}\right)^{-1}$ be the Dedekind zeta-function of $\mathbb{Q}(\eta)$, where $N(\mathfrak{p})$ denotes the norm of a prime ideal $\mathfrak{p}$ of $\mathbb{Q}(\eta)$. Then

$$
H_{F}(s):=\sum_{n \geq 1} h_{F}(n) n^{-s}=G_{F}(s) / \zeta_{F}(s)
$$

where

$$
\begin{aligned}
G_{F}(s):= & \sum_{n \geq 1} b_{F}(n) n^{-s} \\
= & \prod_{p \mid D}\left(1-\varrho_{F}(p) p^{-s}\right) \prod_{p|D \mathfrak{p}| p}\left(1-N(\mathfrak{p})^{-s}\right)^{-1} \\
& \times \prod_{p \nmid D} \prod_{\substack{\mathfrak{p} \mid p \\
f_{\mathfrak{p}}>1}}\left(1-N(\mathfrak{p})^{-s}\right)^{-1} \prod_{p \nmid D}\left(1-\varrho_{F}(p) p^{-s}\right)\left(1-p^{-s}\right)^{-\varrho_{F}(p)}
\end{aligned}
$$

is absolutely convergent in $\operatorname{Re} s>1 / 2$; here $f_{\mathfrak{p}}$ denotes the inertial degree of the prime ideal $\mathfrak{p}$. In particular, for every $\varepsilon>0$

$$
\sum_{\sqrt{x}<n \leq x}\left|b_{F}(n)\right| n^{-1} \ll_{\varepsilon} x^{-1 / 4+\varepsilon} .
$$

Writing $\left(\zeta_{F}(s)\right)^{-1}=\sum_{n \geq 1} a_{F}(n) n^{-s}$, we have (cf. Landau [10], pp. 80-89)

$$
\sum_{n \geq 1} a_{F}(n) n^{-1}=0
$$

and

$$
\sum_{n \leq x} a_{F}(n) \ll x \exp \left(-c(\log x)^{1 / 12}\right)
$$

for some positive constant $c=c(F)$. 
Partial summation, (4.4) and (4.5) yield

$$
\sum_{n \leq x} a_{F}(n) n^{-1} \ll \exp \left(-c_{1}(\log x)^{1 / 12}\right) .
$$

The lemma now follows from (4.3), (4.6) and the identity

$$
\begin{aligned}
\sum_{n \leq x} h_{F}(n) n^{-1}= & \sum_{n \leq \sqrt{x}} b_{F}(n) n^{-1} \sum_{m \leq x / n} a_{F}(m) m^{-1} \\
& +\sum_{\sqrt{x}<n \leq x} b_{F}(n) n^{-1} \sum_{m \leq x / n} a_{F}(m) m^{-1} .
\end{aligned}
$$

Lemma 4.6. For a natural number $M$ let $\omega_{M}$ be a primitive $M$-th root of unity and $\mathbb{Q}_{M}:=\mathbb{Q}\left(\omega_{M}\right)$. If $\mathbb{Q}_{M} \cap K=\mathbb{Q}$, then for integers a relatively prime to $M$ we have

$$
\sum_{\substack{p \leq x \\ p \equiv a(\bmod M)}} \varrho_{F}(p) p^{-1}=\frac{1}{\varphi(M)} \log \log x+O(1) .
$$

Pr o o f. Denote by $\mathcal{G}$ al $(K / \mathbb{Q})$ the Galois group of the extension $K / \mathbb{Q}$ and consider the decomposition $\mathcal{G} a l(K / \mathbb{Q})=\bigcup_{i=1}^{r} \Gamma_{i}$ into conjugation classes. For a rational prime $p$, unramified in $K$, let $\left[\frac{K / \mathbb{Q}}{(p)}\right]$ denote the conjugacy class of the Frobenius automorphism of any prime divisor $\mathfrak{p}$ of $p$. If $D$ is defined as in the proof of Lemma 4.5, then for any $p$ not dividing $D, \varrho_{F}(p)$ depends only upon $\left[\frac{K / \mathbb{Q}}{(p)}\right]$ (cf. [7], Ch. 3, Prop. 2.8), say $\varrho_{F}(p)=\gamma_{i}$ for $\left[\frac{K / \mathbb{Q}}{(p)}\right]=\Gamma_{i}$

By assumption $\operatorname{Gal}\left(K \mathbb{Q}_{M} / \mathbb{Q}\right)=\operatorname{Gal}(K / \mathbb{Q}) \times \mathcal{G}$ al $\left(\mathbb{Q}_{M} / \mathbb{Q}\right)$. If $\tau_{a}$ is the element of $\mathcal{G} a l\left(\mathbb{Q}_{M} / \mathbb{Q}\right)$ such that $\tau_{a}\left(\omega_{M}\right)=\omega_{M}^{a}$, then we have the following decomposition into conjugation classes:

$$
\mathcal{G} a l\left(K \mathbb{Q}_{M} / \mathbb{Q}\right)=\bigcup_{i=1}^{r} \bigcup_{\substack{a(\bmod M) \\(a, M)=1}} \Gamma_{i} \times\left\{\tau_{a}\right\} .
$$

Since $\left[\frac{K \mathbb{Q}_{M} / \mathbb{Q}}{(p)}\right]=\Gamma_{i} \times\left\{\tau_{a}\right\}$ implies $p \equiv a(\bmod M)$ and $\left[\frac{K / \mathbb{Q}}{(p)}\right]=\Gamma_{i}$, that is, $\varrho_{F}(p)=\gamma_{i}$, we have

$$
\sum_{\substack{p \leq x \\ p \equiv a(\bmod M)}} \varrho_{F}(p)=\sum_{i=1}^{r} \gamma_{i} \cdot \pi_{(i, a)}(x)+O(1),
$$


where $\pi_{(i, a)}(x)$ is the number of primes $p$ not exceeding $x$ for which $\left[\frac{K \mathbb{Q}_{M} / \mathbb{Q}}{(p)}\right]=\Gamma_{i} \times\left\{\tau_{a}\right\}$.

By Chebotarev's density theorem with error term (cf. [9]), (4.8) implies that

$$
\sum_{\substack{p \leq x \\ p \equiv a(\bmod M)}} \varrho_{F}(p)=\lambda \cdot \operatorname{li}(x)+O(x \exp (-c \sqrt{\log x})),
$$

where the constant

$$
\lambda:=\left[K \mathbb{Q}_{M}: \mathbb{Q}\right]^{-1} \cdot \sum_{i=1}^{r}\left|\Gamma_{i}\right| \gamma_{i}
$$

is independent of $a$. Partial summation in (4.9), gives

$$
\sum_{\substack{p \leq x \\ p \equiv a(\bmod M)}} \varrho_{F}(p) p^{-1}=\lambda \log \log x+O(1),
$$

and a comparison with (4.2) yields $\lambda=1 / \varphi(M)$, which proves (4.7).

Using the previous two lemmas we can now easily prove

TheOREM 4.7. For an irreducible nonconstant polynomial $F \in \mathbb{Z}[x]$ let

$$
\varphi_{F}(n):=n \prod_{p \mid n}\left(1-\varrho_{F}(p) / p\right),
$$

where $\varrho_{F}(p)$ is the number of zeros of $F(\bmod p)$. If

$$
c_{F}:=\frac{1}{2} \prod_{p}\left(1-\varrho_{F}(p) p^{-2}\right)
$$

and $q$ denotes the smallest odd prime that is unramified in a splitting field $K$ of $F(x)$, then

$$
\sum_{n \leq x} \varphi_{F}(n)=c_{F} x^{2}+R_{F}(x)
$$

where

$$
R_{F}(x) \ll x \log x
$$

and

$$
R_{F}(x)=\Omega_{ \pm}\left(x(\log \log x)^{1 /(q-1)}\right) .
$$

Proof. Recall that $\varphi_{F}=I * h_{F}$ with $h_{F} \in \mathcal{C}(0,1)$. By Lemma 4.5 there are positive constants $c_{1}$ and $c_{2}$ such that

$$
\sup _{y>x}\left|\sum_{x<n \leq y} h_{F}(n) n^{-1}\right| \leq c_{1} \exp \left(-c_{2}(\log (1+x))^{1 / 12}\right)=: \xi_{F}(x) .
$$

Obviously $\xi_{F}$ satisfies the assumptions of Corollary 2.3. 
Since $q$ is totally ramified in $\mathbb{Q}_{q}$, we have $\mathbb{Q}_{q} \cap K=\mathbb{Q}$. Lemma 4.6 and formula (4.2) show that

$$
\sum_{\substack{p \leq x \\ p \equiv a(\bmod q)}} \varrho_{F}(p) p^{-1}=\frac{1}{\varphi(q)} \Theta_{F}(x)+O(1)=\frac{1}{q-1} \log \log x+O(1) .
$$

An application of Corollary 2.3 yields the proof.

Up to this point our examples have dealt with functions $I * h$, where $h \in \mathcal{C}(r, 1)$ for some nonnegative $r$. In closing we will therefore bring an application of Corollary 2.3 which involves the class $\mathcal{C}(0,2)$. The relevant function $f$ is defined by

$$
f(n):=\sum_{\substack{d \mid n \\(d, n / d)=1}} \varphi(d) ;
$$

$f(n)$ is the number of integers possessing weak order $(\bmod n)(\mathrm{cf} .[8])$. In this case $f=I * h$ where

$$
h\left(p^{\alpha}\right):= \begin{cases}0 & \text { if } \alpha=1 \\ 1-p & \text { if } \alpha \geq 2 .\end{cases}
$$

It can be seen without too much difficulty that $h \in \mathcal{C}(0,2)$ and it can be shown that

$$
\sup _{y>x}\left|\sum_{x<n \leq y} h(n) n^{-1}\right| \ll \exp (-c \sqrt{\log x})
$$

(cf. [6]). Hence Corollary 2.3 gives

$$
\sum_{n \leq x} f(n)=\left(\frac{1}{2} \sum_{n \geq 1} h(n) n^{-2}\right) x^{2}+R(x)
$$

where $R(x) \ll x \log x$ and $R(x)=\Omega_{ \pm}(x \sqrt{\log \log x})$.

\section{References}

[1] T. M. Apostol, Introduction to Analytic Number Theory, Springer, New York 1976.

[2] R. Dedekind, Gesammelte mathematische Werke. Erster Band, R. Fricke, E. Noether and Ö. Ore (eds.), Vieweg, Braunschweig 1930.

[3] P. Erdős, On the sum $\sum_{k<x} d(f(k))$, J. London Math. Soc. 27 (1952), 7-15.

[4] P. Erdös and H. N. Shapiro, On the changes of sign of a certain error function, Canad. J. Math. 3 (1951), 375-385.

[5] H. Halberstam and H.-E. Richert, On a result of R. R. Hall, J. Number Theory 11 (1979), 76-89.

[6] J. Herzog and P. R. Smith, Asymptotic results on the distribution of integers possessing weak order $(\bmod m)$, preprint, Frankfurt 1990.

[7] G. J. Janusz, Algebraic Number Fields, Academic Press, New York 1973. 
[8] V.S. Joshi, Order free integers $(\bmod m)$, in: Number Theory, Mysore 1981, Lecture Notes in Math. 938, Springer, New York 1982, 93-100.

[9] J. C. Lagarias and A. M. Odlyzko, Effective versions of the Chebotarev density theorem, in: Algebraic Number Fields: $L$-functions and Galois Properties, Proc. Sympos. Durham 1975, Academic Press, London 1977, 409-464.

[10] E. Landau, Über die zahlentheoretische Funktion $\mu(k)$, in: Collected Works, Vol. 2, L. Mirsky et al. (eds.), Thales Verlag, Essen 1986, 60-93.

[11] —, Vorlesungen über Zahlentheorie, Chelsea, New York 1950.

[12] F. Mertens, Über einige asymptotische Gesetze der Zahlentheorie, J. Reine Angew. Math. 77 (1874), 289-338.

[13] H. L. Montgomery, Fluctuations in the mean of Euler's phi function, Proc. Indian Acad. Sci. (Math. Sci.) 97 (1987), 239-245.

[14] S. S. Pillai and S. D. Chowla, On the error terms in some asymptotic formulae in the theory of numbers (I), J. London Math. Soc. 5 (1930), 95-101.

[15] J. H. Proschan, On the changes of sign of a certain class of error functions, Acta Arith. 17 (1971), 407-430.

[16] H. Stevens, Generalizations of the Euler $\varphi$-function, Duke Math. J. 38 (1971), $181-186$.

[17] A. Walfisz, Weylsche Exponentialsummen in der neueren Zahlentheorie, Deutsch. Verlag Wiss., Berlin 1963.

J. W. GOETHE-UNIVERSITÄT

FACHBEREICH MATHEMATIK

ROBERT-MAYER-STR. 6-10

D-6000 FRANKFURT AM MAIN

FEDERAL REPUBLIC OF GERMANY 